\title{
Perfil epidemiológico da sífilis congênita em gestantes no município de Maceió
}

\author{
Epidemiological profile of congenital syphilis in pregnant women in the municipality of \\ Maceió
}

Perfil epidemiológico de la sífilis congénita en mujeres embarazadas en el municipio de Maceió

Douglas Ferreira Rocha Barbosa ${ }^{1 *}$, Aline Cynara Coelho Albuquerque ${ }^{1}$, Rosa Caroline Mata Verçosa ${ }^{1}$, Rosane Pereira dos Reis ${ }^{1}$, Ediane Gonçalves ${ }^{1}$, Layanne Ramalho Jacob ${ }^{2}$, Kleytonn Giann Silva de Santana ${ }^{4}$, Sidlayne dos Santos ${ }^{3}$, Thalita da Silva Pereira ${ }^{1}$, Margarete Batista da Silva ${ }^{5}$.

\section{RESUMO}

Objetivo: Analisar o perfil epidemiológico da sífilis congênita em gestantes no município de Maceió/AL. Métodos: Trata-se de um estudo epidemiológico caracterizado como descritivo, observacional, retrospectivo e transversal, sendo realizado a partir de dados coletados do Sistema de Informações de Agravos de Notificações de dados de sífilis congênita em gestantes no período de 2014-2019. Resultado e discussão: Embora a sífilis seja uma doença com altos índices de infecção entre as gestantes, poucas possuem informações sobre a doença, seu modo de transmissão, características, diagnóstico e tratamento. Os resultados evidenciados nesse estudo revelam que o perfil da sífilis congênita e materna no município de Maceió encontra-se com número de casos ainda alto. Conclusão: Os achados desse estudo permitem concluir que o perfil epidemiológico da sífilis congênita em gestantes no município de Maceió ainda encontrase com um elevado número de casos com 115 casos no ano de 2019, diante disso se faz necessário à busca ativa de gestantes para realização do pré-natal, e reforçar os exames clínicos entre as que já o realizam.

Palavras-chave: Epidemiologia, Sífilis, Doenças sexualmente transmissíveis.

\begin{abstract}
Objective: To analyze the epidemiological profile of congenital syphilis in pregnant women in the city of Maceió/AL. Methods: This is an epidemiological study characterized as descriptive, observational, retrospective and cross-sectional, being carried out from data collected from the Information System for Diseases of Congenital Syphilis Data in pregnant women in the period 2014-2019. Results and discussion: Although syphilis is a disease with high rates of infection among pregnant women, few have information about the disease, its mode of transmission, characteristics, diagnosis and treatment. The results evidenced in this study reveal that the profile of congenital and maternal syphilis in the city of Maceió is still with a high number of cases. Conclusion: The findings of this study allow us to conclude that the epidemiological profile of congenital syphilis in pregnant women in the city of Maceió is still with a high number of cases with 115 cases in 2019, therefore it is necessary to actively search for pregnant women to perform the procedure, prenatal care, and reinforce clinical examinations among those who already perform it.
\end{abstract}

Keywords: Epidemiology, Syphilis, Sexually transmitted diseases.

\footnotetext{
${ }^{1}$ Faculdade Estácio de Alagoas, Maceió - AL. *E-mail: douglasrochaefata@hotmail.com

2 Universidade Estadual de Ciências da Saúde de Alagoas (UNCISAL), Maceió - AL.

${ }^{3}$ Secretaria Municipal de Matriz de Camaragibe, Matriz de Camaragibe - AL.

${ }^{4}$ Instituto de Medicina Integral Professor Fernando Figueira, Recife - PE.

${ }^{5}$ Centro Universitário Tiradentes, Maceió - AL.
} 


\section{RESUMEN}

Objetivo: Analizar el perfil epidemiológico de la sífilis congénita en mujeres embarazadas en la ciudad de Maceió/AL. Métodos: Este es un estudio epidemiológico caracterizado como descriptivo, observacional, retrospectivo y transversal, que se lleva a cabo a partir de datos recopilados del Sistema de Información para Enfermedades de Sífilis Congénita en mujeres embarazadas en el período de 2014-2019. Resultados y discusión: Aunque la sífilis es una enfermedad con altas tasas de infección entre las mujeres embarazadas, pocas tienen información sobre la enfermedad, su modo de transmisión, características, diagnóstico y tratamiento. Los resultados evidenciados en este estudio revelan que el perfil de sífilis congénita y materna en la ciudad de Maceió todavía presenta una gran cantidad de casos. Conclusión: Los hallazgos de este estudio permiten concluir que el perfil epidemiológico de la sífilis congénita en gestantes en la ciudad de Maceió aún presenta un elevado número de casos con 115 casos en 2019, por lo que es necesario buscar activamente gestantes para realizar el procedimiento. atención prenatal, y reforzar los exámenes clínicos entre quienes ya la realizan.

Palabras clave: Epidemiología, Sífilis, Enfermedades de transmisión sexual.

\section{INTRODUÇÃO}

A sífilis é uma doença infecciosa que tem como agente etiológico a bactéria Treponema pallidum. É uma patologia de caráter sistêmico, prevenível, porém quando não tratada, evolui para um quadro crônico com sequelas irreversíveis, podendo ser transmitida por via sexual e vertical, raramente por transfusão sanguínea (BRASIL, 2017).

Atualmente, a sífilis é uma preocupação de grande parte dos países, e na tentativa de apresentar novas estratégias para o seu controle, a Organização Mundial de Saúde (OMS), juntamente com a Organização Pan-americana de Saúde (OPAS), lançou uma proposta, adotada pelo Ministério da Saúde (MS) do Brasil, com objetivo global de eliminar a sífilis congênita como um problema de saúde pública, sendo aceitável uma incidência de até 0,5 casos por mil nascidos vivos (BRASIL, 2015a).

No Brasil, estima-se que entre os anos 2005-2014, foram notificados 100.790 casos de sífilis gestacional (SG) e 104.853 de sífilis congênita (SC). Apenas no ano de 2013, foram notificados 21.382 casos de SG e 13.705 de SC. Nos últimos anos houve um aumento na taxa da doença, sendo no ano de 2013 tiveram 4,7 e 7,4 casos por mil nascidos vivos (BRASIL, 2015b).

A SC está geralmente associada às gestantes que não realizaram o pré-natal e/ou aquelas que não realizaram o tratamento, ou o mesmo foi inadequado, podendo resultar na morte fetal, morte neonatal e na prematuridade (PADOVANI C, et al., 2018).

Nesse contexto, o MS lançou o Protocolo Clínico e Diretrizes Terapêuticas para Prevenção da Transmissão Vertical de HIV, Sífilis e Hepatites Virais (PCDT-TV) que se propõe a ser uma ferramenta de linguagem objetiva, de modo a facilitar o acesso às principais informações e recomendações, possibilitando a sua utilização pela equipe multiprofissional de saúde no cuidado integral às gestantes, suas parcerias sexuais e crianças expostas (BRASIL, 2018). No Brasil, a infecção da sífilis congênita é de notificação compulsória conforme Portaria oㅡ 542/1986 do MS, e a gestacional segundo a Portaria no 33 de 14 de julho de 2005 (BOTTURA BR, et al., 2019).

A notificação da sífilis adquirida, sífilis gestacional e congênita é obrigatória conforme portaria do Ministério da Saúde e a testagem deve ser realizados pela gestante para prevenir a transmissão vertical na primeira consulta do pré-natal (idealmente, no primeiro trimestre da gestação), no início do terceiro trimestre (28 semana) e no momento do parto ou aborto, independentemente de exames anteriores (BRASIL, 2018).

Há evidências científicas da ocorrência da sífilis congênita relacionada às fragilidades dos serviços de saúde quanto à cobertura e assistência do pré-natal na atenção básica. Ademais, as características maternas também vêm se associando à ocorrência da sífilis congênita. Em estudo de base nacional foi identificado associação dos casos de sífilis congênita em mulheres na faixa etária de 20 a 34 anos, com a menor 
escolaridade, cor da pele preta, sem atividade remunerada, início tardio do pré-natal e menor número de consultas e exames sorológicos, tratamento inadequado. Além disso, a sífilis congênita configura-se como um fator de risco para a prematuridade, mortalidade fetal e frequência de internações hospitalares (BRASIL, 2015a).

Para diagnosticar a sífilis gestacional, são utilizados comumente os testes treponêmicos rápidos ou os testes treponêmicos convencionais (Elisa, FTA-Abs, TPHA) e também os não treponêmicos (VDRL, RPR, TRUST) (BRASIL, 2016). Vale destacar que o teste rápido é uma importante estratégia para esse diagnóstico, podendo qualificar o atendimento já que não requer tecnologias complexas, sendo de rápido resultado, com média de 30 minutos, agilizando a adoção de medidas para prevenção e tratamento (BRASIL, 2012).

Para o diagnóstico da sífilis congênita, deve-se avaliar a história clínico-epidemiológica da mãe, realizar exame físico detalhado da criança e avaliar os resultados dos testes laboratoriais e dos exames radiológicos (BRASIL, 2016). A busca pela confirmação de SC será considerada nas situações: toda criança nascida de mãe portadora da sífilis e com evidência clínica e/ou laboratorial, tendo o seu diagnóstico durante a gestação, parto ou puerpério. E também, todo indivíduo menor de 13 anos de idade e com suspeita clínica e/ou epidemiológica da sífilis congênita (BRASIL, 2006).

A Atenção Básica $(A B)$ é fundamental no combate à sífilis gestacional e congênita, pois é a porta de entrada para os serviços de saúde. Os profissionais que atuam diretamente com as gestantes na $A B$, necessitam de mais preparo técnico e uma visão interdisciplinar pela preocupante complexidade e agravo da doença (CAVALCANTE PAM, et al., 2017).

Esse trabalho mostra-se relevante para que os profissionais de saúde em geral, em especialmente para o enfermeiro, pois está mais próximo da gestante, realizando consultas de pré-natal, buscando estratégias para identificar os casos de sífilis e orienta as gestantes e seus parceiros quanto aos riscos das infecções sexualmente transmissíveis.

Diante do exposto, este trabalho traz como pergunta da pesquisa: qual o perfil epidemiológico da sífilis em gestante e congênita no município de Maceió/AL? Considerando o impacto da sífilis gestacional e congênita na saúde pública e a necessidade de seu controle, este estudo tem como objetivo: analisar o perfil epidemiológico da sífilis gestacional e congênita no município de Maceió/AL.

\section{MÉTODOS}

Trata-se de um estudo epidemiológico caracterizado como descritivo, observacional, retrospectivo e transversal, sendo realizado a partir de dados coletados do Sistema de Informações de Agravos de Notificações (SINAN), disponibilizados pelo Departamento de Doenças de Condições Crônicas e Infecções Sexualmente Transmissíveis, através da página indicadoressifilis.aids.gov.br no período de janeiro a julho de 2020.

Foram incluídos no estudo todos os casos de sífilis congênita e gestacional notificados no município de Maceió/AL, entre 2014-2019. Foi utilizado como critério de exclusão os dados que se encontravam incompletos.

Para organização e tabulação dos dados foi utilizado o programa Microsoft $\circledast$ Excel® 2010, com a finalidade de averiguar os aspectos relevantes da pesquisa. Vale destacar que de acordo com a Resolução no 510/2016 do Conselho Nacional de Saúde, por ser um sistema de domínio público e não passível de identificação dos sujeitos, não houve necessidade de apreciação em Comitê de Ética em Pesquisa (CEP) desse estudo.

\section{RESULTADOS}

Nos resultados desse estudo serão apresentados números de casos e taxa de detecção por 1.000 nascidos vivos. Além disso, serão apresentados casos e percentual de gestantes com sífilis por ano de diagnóstico segundo idade gestacional, faixa etária, escolaridade, raça e cor e seu esquema de tratamento prescrito. 
Também serão apresentados números e distribuição percentual segundo idade da criança, números e distribuição percentual de sífilis congênita segundo diagnóstico final, informação sobre realização de pré-natal da mãe, esquema de tratamento da mãe e do parceiro.

Foram destacados dados sobre a sífilis gestacional e congênita, nela observa-se o crescente número de casos e da taxa de deteç̧ão nos anos de 2014 a 2019. Sendo possível observar que a partir do ano de 2015 o número de casos aumentou substancialmente, passando de 78 para 409 casos em 2018, reduzindo para 115 em 2019 (Tabela 1).

Tabela 1 - Número de casos e taxa de detecção por 1.000 nascidos vivos de gestantes com sífilis por ano de diagnóstico.

\begin{tabular}{ccccccc}
\hline Maceió $-\mathbf{A L}$ & $\mathbf{2 0 1 4}$ & $\mathbf{2 0 1 5}$ & $\mathbf{2 0 1 6}$ & $\mathbf{2 0 1 7}$ & $\mathbf{2 0 1 8}$ & $\mathbf{2 0 1 9}$ \\
\hline Casos & 78 & 78 & 121 & 249 & 409 & 115 \\
Taxa de Detecção & 5,2 & 5,0 & 8,6 & 17,1 & 28,1 & - \\
\hline
\end{tabular}

Fonte: Barbosa DFR, et al., 2020. Dados coletados de MS/SVS/DCCI - Departamento de Doenças de Condições Crônicas e Infecções Sexualmente Transmissíveis, 2020.

A detecção da sífilis em gestantes é ignorada no início da gestação, sendo frequentemente detectada a partir do $3^{\circ}$ trimestre gestacional, quando analisada a idade gestacional de detecção da sífilis, foi observado em 2018 que (25,7\%) das gestantes foram diagnosticadas ainda no primeiro trimestre, além disso, 36,7\% tiveram seu diagnóstico realizado no segundo trimestre e $35 \%$ no terceiro, já na faixa etária de gestantes com sífilis, o maior número de casos vem sendo mulheres de 20 a 29 anos, seguidas pela faixa etária de 15 a 19 anos.

Em relação à variável idade materna, observa-se uma predominância na faixa etária de 20 a 29 anos que, em 2019, apresentaram 50 (43,5\%) casos, seguida a faixa de 15 a 19 anos com 41 (35,7\%) casos. Com relação à escolaridade, observou-se um aumento no percentual de casos em indivíduos analfabetos ou com ensino fundamental incompleto comparando com mulheres com ensino superior.

Quanto à raça/cor, observou-se que os números de diagnósticos em mulheres de cor parda sempre foram predominantes em relação às mulheres brancas e pretas. Em toda a série histórica, a notificação de indivíduos de raça/cor amarela e indígena separadamente sempre se mostrou com índices baixos.

O esquema de tratamento mais utilizado entre as gestantes com sífilis é a penicilina. Deve-se atentar para o alto índice de mulheres que não realizam qualquer esquema de tratamento $(4,4 \%)$ ou de casos ignorados (5,9\%) no ano de 2018. (Tabela 2).

A detecção da doença é maior em crianças com idade menor que 7 dias, seguido por crianças com 28 a 364 dias de vida, um aumento considerável nos últimos anos de casos de sífilis congênita recente, cerca de $97,4 \%$ de casos no ano de 2019 comparando com 2014 com 89,1\% dos casos. Quanto às características da assistência pré-natal, verifica-se que houve uma diminuição dos casos em 2019 em gestantes que realizou o pré-natal e tiveram diagnóstico da sífilis congênita 74 (63,8\%) casos.

Ressaltando um índice alto de casos ignorados $(28,4 \%)$, os casos de tratamentos ignorados para sífilis congênita lideram a porcentagem com $43,1 \%$, seguindo dos tratamentos inadequados com $37,1 \%$. Entre os parceiros que não seguem tratamento são superiores aos que realizam tratamento para sífilis em todos os anos estudados (Tabela 3). 
Tabela 2 - Números e distribuição percentual de casos gestantes com sífilis segundo a idade gestacional, faixa etária, escolaridade, raça/cor e esquema de tratamento prescrito por ano de diagnóstico.

\begin{tabular}{|c|c|c|c|c|c|c|}
\hline \multirow{2}{*}{ Maceió - AL } & 2014 & 2015 & 2016 & 2017 & 2018 & \multirow{2}{*}{$\begin{array}{l}2019 \\
\mathrm{n} / \%\end{array}$} \\
\hline & n / \% & n / \% & $\mathrm{n} / \%$ & n / \% & n / \% & \\
\hline \multicolumn{7}{|l|}{$\begin{array}{c}\text { Idade } \\
\text { Gestacional }\end{array}$} \\
\hline 1을 Trimestre & $1(19,2)$ & $11(14,1)$ & $35(28,9)$ & $60(24,1)$ & $105(25,7)$ & $2(18,3)$ \\
\hline $2^{\circ}$ Trimestre & $27(34,3)$ & $35(44,9)$ & $55(45,5)$ & $91(36,5)$ & $150(36,7)$ & $35(30,4)$ \\
\hline $3^{\circ}$ Trimestre & $35(44,9)$ & $30(38,5)$ & $31(25,6)$ & $87(34,9)$ & $146(35,0)$ & $55(47,8)$ \\
\hline Ignorada & $1(1,3)$ & $2(2,6)$ & $(-)$ & $11(4,4)$ & $11(2,7)$ & $4(3,5)$ \\
\hline \multicolumn{7}{|l|}{ Faixa Etária } \\
\hline 10 a 14 Anos & $2(2,6)$ & $4(5,1)$ & $2(1,7)$ & $5(2,0)$ & $6(1,5)$ & $10(0,9)$ \\
\hline 15 a 19 Anos & $1(32,1)$ & $24(1,8)$ & $48(39,7)$ & $60(24,1)$ & $110(26,9)$ & $41(35,7)$ \\
\hline 20 a 29 Anos & $33(42,3)$ & $37(47,4)$ & $55(45,5)$ & $142(57,0)$ & $206(50,4)$ & $50(43,5)$ \\
\hline 30 a 39 Anos & $17(-)$ & $10(-)$ & $14(-)$ & $39(-)$ & $76(-)$ & $21(-)$ \\
\hline $\begin{array}{c}40 \text { Anos ou } \\
\text { Mais }\end{array}$ & $5(-)$ & $3(-)$ & $2(-)$ & $3(-)$ & $1(-)$ & $2(-)$ \\
\hline Ignorado & $(-)$ & $(-)$ & $(-)$ & $(-)$ & $(-)$ & $(-)$ \\
\hline \multicolumn{7}{|l|}{ Escolaridade } \\
\hline Analfabeto & $1(1,3)$ & $5(6,4)$ & $3(2,5)$ & $3(1,2)$ & $8(2,0)$ & $(-)$ \\
\hline $\begin{array}{l}1^{\text {a }} 4^{\text {a }} \text { Série } \\
\text { Incompleta }\end{array}$ & $14(17,9)$ & $7(9,0)$ & $9(7,4)$ & $22(8,8)$ & $27(6,6)$ & $4(3,5)$ \\
\hline $\begin{array}{l}4^{\text {a }} \text { Série } \\
\text { Completa }\end{array}$ & $5(6,4)$ & $4(5,1)$ & $6(5,0)$ & $13(5,2)$ & $13(3,2)$ & $3(2,6)$ \\
\hline $\begin{array}{l}5^{a} 8^{a} \text { Série } \\
\text { Incompleta }\end{array}$ & $28(3,5)$ & $21(26,9)$ & $27(22,3)$ & $76(30,5)$ & $128(31,3)$ & $30(26,1)$ \\
\hline $\begin{array}{l}\text { Fundamental } \\
\text { Completo }\end{array}$ & $4(5,1)$ & $8(10,3)$ & $9(7,4)$ & $13(5,2)$ & $16(3,9)$ & $9(7,8)$ \\
\hline $\begin{array}{c}\text { Médio } \\
\text { Incompleto }\end{array}$ & $2(2,6)$ & $8(10,3)$ & $19(15,7)$ & $26(10,4)$ & $48(11,7)$ & $19(16,5)$ \\
\hline $\begin{array}{l}\text { Médio } \\
\text { Completo }\end{array}$ & $7(9,0)$ & $7(9,0)$ & $11(9,1)$ & $30(12,0)$ & $48(11,7)$ & $11(7,0)$ \\
\hline $\begin{array}{l}\text { Superior } \\
\text { Incompleto }\end{array}$ & $(-)$ & $1(1,3)$ & $1(0,8)$ & $2(0,8)$ & $3(0,7)$ & $2(1,7)$ \\
\hline $\begin{array}{l}\text { Superior } \\
\text { Completo }\end{array}$ & $(-)$ & $1(1,3)$ & $2(1,7)$ & $5(2,0)$ & $3(0,7)$ & $(-)$ \\
\hline $\begin{array}{l}\text { Não Se } \\
\text { Aplica }\end{array}$ & $(-)$ & $(-)$ & $(-)$ & $(-)$ & $(-)$ & $(-)$ \\
\hline Ignorado & $17(21,8)$ & $16(20,5)$ & $34(28,1)$ & $59(23,7)$ & $115(28,1)$ & $40(34,8)$ \\
\hline Analfabeto & $1(1,3)$ & $5(6,4)$ & $3(2,5)$ & $3(1,2)$ & $8(2,0)$ & $(-)$ \\
\hline \multicolumn{7}{|l|}{ Raça/Cor } \\
\hline Branca & $10(12,8)$ & $5(6,4)$ & $20(16,5)$ & $25(10,0)$ & $38(9,3)$ & $16(13,9)$ \\
\hline Preta & $12(15,4)$ & $19(24,4)$ & $20(16,5)$ & $28(11,2)$ & $46(11,2)$ & $12(10,3)$ \\
\hline Amarela & $1(1,3)$ & $4(5,1)$ & $2(1,7)$ & $4(1,6)$ & $8(2,0)$ & $1(0,9)$ \\
\hline Parda & $53(67,9)$ & $46(59,0)$ & $73(60,3)$ & $180(73,3)$ & $276(67,5)$ & $68(59,1)$ \\
\hline Indígena & $(-)$ & $1(1,3)$ & $(-)$ & $(-)$ & $(-)$ & $(-)$ \\
\hline Ignorada & $2(2,6)$ & $3(3,8)$ & $6(5,0)$ & $12(4,8)$ & $41(10,0)$ & $18(15,7)$ \\
\hline \multicolumn{7}{|l|}{$\begin{array}{c}\text { Esquema de } \\
\text { Tratamento } \\
\text { Prescrito }\end{array}$} \\
\hline Penicilina & $(-)$ & $66(84,6)$ & $100(82,6)$ & $211(84,7)$ & $356(87,0)$ & $(-)$ \\
\hline $\begin{array}{c}\text { Outro } \\
\text { Esquema }\end{array}$ & $(-)$ & $4(5,1)$ & $(-)$ & $1(0,4)$ & $11(2,7)$ & $(-)$ \\
\hline $\begin{array}{l}\text { Não realizado } \\
\text { Ignorado }\end{array}$ & $\begin{array}{l}(-) \\
(-)\end{array}$ & $\begin{array}{l}3(3,8) \\
5(6,4)\end{array}$ & $\begin{array}{c}4(3,3) \\
17(4,0)\end{array}$ & $\begin{array}{c}7(2,8) \\
30(12,0)\end{array}$ & $\begin{array}{l}18(4,4) \\
24(5,9)\end{array}$ & $\begin{array}{l}(-) \\
(-)\end{array}$ \\
\hline
\end{tabular}

Fonte: Barbosa DFR, et al., 2020. Dados coletados de MS/SVS/DCCI - Departamento de Doenças de Condições Crônicas e Infecções Sexualmente Transmissíveis, 2020. 
Tabela 3 - Números e distribuição percentual de sífilis congênita segundo a idade da criança, diagnóstico final, realização do pré-natal, esquema de tratamento da mãe e tratamento do parceiro da mãe.

\begin{tabular}{|c|c|c|c|c|c|c|}
\hline \multirow{2}{*}{ Maceió - AL } & 2014 & 2015 & 2016 & 2017 & 2018 & 2019 \\
\hline & n / \% & n / \% & n / \% & n / \% & n / \% & n / \% \\
\hline \multicolumn{7}{|l|}{ Idade da Criança } \\
\hline Menos de 7 Dias & $21(97,3)$ & $185(96,4)$ & 14392,3 & 16995,5 & 19288,9 & 10187,1 \\
\hline 7 a 27 Dias & $1(0,5)$ & $3(1,6)$ & $10(6,5)$ & $5(2,8)$ & $22(10,2)$ & $12(10,3)$ \\
\hline 28 a 364 Dias & $5(2,3)$ & $4(2,1)$ & $2(1,3)$ & $1(1,3)$ & $2(0,9)$ & $2(1,7)$ \\
\hline 1 Ano & $(-)$ & $(-)$ & $(-)$ & $2(1,1)$ & $(-)$ & $1(0,9)$ \\
\hline 2 a 4 Anos & $(-)$ & $(-)$ & $(-)$ & $(-)$ & $(-)$ & $(-)$ \\
\hline 5 a 12 Anos & $(-)$ & $(-)$ & $(-)$ & $(-)$ & $(-)$ & $(-)$ \\
\hline Ignorado & $(-)$ & $(-)$ & $(-)$ & $(-)$ & $(-)$ & $(-)$ \\
\hline \multicolumn{7}{|l|}{$\begin{array}{c}\text { Diagnóstico } \\
\text { Final }\end{array}$} \\
\hline $\begin{array}{l}\text { Sífilis Congênita } \\
\text { Recente }\end{array}$ & $196(89,1)$ & $166(86,5)$ & 14090,3 & 16794,4 & 21197,7 & 11397,4 \\
\hline $\begin{array}{c}\text { Sífilis Congênita } \\
\text { Tardia }\end{array}$ & 0 & 0 & 0 & 0 & 0 & 0 \\
\hline Aborto por Sífilis & $10(4,5)$ & $15(7,8)$ & $8(5,2)$ & $3(1,7)$ & $3(1,4)$ & $1(0,9)$ \\
\hline $\begin{array}{l}\text { Natimorto por } \\
\text { Sífilis }\end{array}$ & $14(6,4)$ & $11(5,7)$ & $7(4,5)$ & $7(4,0)$ & $2(0,9)$ & $2(1,7)$ \\
\hline \multicolumn{7}{|l|}{$\begin{array}{l}\text { Realização do } \\
\text { Pré-Natal }\end{array}$} \\
\hline Sim & $163(74,1)$ & $141(73,4)$ & $114(73,5)$ & $133(75,1)$ & $163(75,5)$ & $74(63,8)$ \\
\hline Não & $41(18,6)$ & $43(22,4)$ & $28(18,1)$ & $39(22,0)$ & $24(11,1)$ & $9(7,8)$ \\
\hline Ignorado & $16(7,3)$ & $8(4,2)$ & $13(8,4)$ & $5(2,8)$ & $29(13,4)$ & $33(28,4)$ \\
\hline \multicolumn{7}{|l|}{$\begin{array}{l}\text { Esquema de } \\
\text { Tratamento da } \\
\text { Mãe }\end{array}$} \\
\hline Adequado & $1(0,5)$ & $3(1,6)$ & $(-)$ & $3(1,7)$ & $8(3,7)$ & $2(1,7)$ \\
\hline Inadequado & $87(39,5)$ & $70(36,5)$ & $74(47,7)$ & $86(48,6)$ & $36(63,0)$ & $43(37,1)$ \\
\hline Não realizado & $114(51,8)$ & $112(58,3)$ & $72(46,5)$ & $83(46,9)$ & $36(16,7)$ & $21(18,1)$ \\
\hline Ignorado & $18(8,2)$ & $7(3,6)$ & $9(5,8)$ & $5(2,8)$ & $36(16,7)$ & $50(43,1)$ \\
\hline
\end{tabular}

Tratamento do

Parceiro da Mãe

\begin{tabular}{ccccccc} 
Sim & $16(7,3)$ & $14(7,3)$ & $15(9,7)$ & $12(6,8)$ & $20(9,3)$ & $7(6,0)$ \\
Não & $151(68,6)$ & $155(80,7)$ & $116(74,8)$ & $136(76,8)$ & $146(67,6)$ & $72(62,1)$ \\
Ignorado & $53(24,1)$ & $23(12,0)$ & $24(15,5)$ & $29(16,4)$ & $50(23,1)$ & $37(31,9)$ \\
\hline
\end{tabular}

Fonte: Barbosa DFR, et al., 2020. Dados coletados de MS/SVS/DCCl - Departamento de Doenças de Condições Crônicas e Infecções Sexualmente Transmissíveis, 2020.

\section{DISCUSSÃO}

A sífilis é uma doença ainda reemergente no Brasil e o seu aumento é visível em todas as regiões brasileiras, o que confirma a necessidade do desenvolvimento de mais ações voltadas para o seu controle (BOTTURA BR, et al., 2019).

Com o controle da sífilis, é possível evitar os casos de transmissão vertical da doença, ou seja, de mãe para feto e assim poder oferecer uma maior chance de cura para a mãe evitando que o bebê ao nascer desenvolva maiores complicações como a prematuridade, baixo peso, danos neurológicos, comprometimento auditivo e/ou oftalmológico (DE AQUINO GT, et al., 2016). 
Os resultados desse estudo revelam a importância do acompanhamento das gestantes no pré-natal e o rastreamento desde o primeiro contato do profissional da saúde com a mulher. Embora a sífilis seja uma doença com altos índices de infecção entre as gestantes, poucas possuem informações sobre a doença, seu modo de transmissão, características, diagnóstico e tratamento. Segundo Neto LG, et al. (2019), apesar da sífilis ser uma doença de fácil tratamento e prevenção, os números de casos revelam um aumento significativo da incidência dessa infecção entre as gestantes. Sendo uma das causas para o aumento dos números de casos, o aumento da quantidade de testes-rápido no pré-natal e a redução do uso do preservativo na prática sexual.

Essa pesquisa evidencia o aumento progressivo da taxa de detecção entre 1.000 nascidos vivos de gestantes com sífilis, nos anos de 2014 a 2018 e o aumento de casos entre os anos de 2014 a 2019, tendo uma considerável queda a partir do ano de 2019. O número crescente da sífilis no município de Maceió é progressivo, quando comparamos as taxas de detecção da doença em relação ao número de casos no Brasil. Estudos mostram que a região que apresenta maior taxa de detecção é a região Sudeste, sendo provável por apresentar uma população mais numerosa e maior número de subnotificações quando comparamos a outras regiões brasileiras (BOTTURA BR, et al., 2019).

No presente estudo é observado, segundo a idade gestacional, que as gestantes que se encontravam no seu $3^{\circ}$ trimestre de gestação no ano de 2019 é o maior número de casos, representando 47,8\%, em comparação aos demais períodos de gestação desse ano. Em contraponto, a menor taxa de detecção estava em mulheres com gestação de $1^{\circ}$ trimestre, no qual foi detectado $2(18,3 \%)$ casos. Sendo visível que no município de Maceió o diagnóstico é tardio. Já no estudo de Lima TM, et al. (2019), avalia-se que, entre os anos de 2007 a 2016, 41,16\% dos casos são de mulheres diagnosticadas no $1^{\circ}$ trimestre da gestação.

Segundo a faixa etária, a taxa de sífilis gestacional entre os anos de 2014 e 2019, é maior entre mulheres de 20 a 29 anos, sendo seu pico de casos no ano de 2018, no qual os resultados mostram um número de $206(50,4 \%)$ casos em relação às outras faixas etárias do mesmo ano. Em 2019 foi observada uma queda em todas as faixas etárias em comparação com os três últimos anos.

Quando comparada a outras localidades brasileiras, como no estado de Minas Gerais, a faixa etária da sífilis gestacional entre mulheres de 20 a 34 anos é a maior, com cerca de $64,8 \%$ dos casos entre os anos de 2007 a 2017 (GOMES FT, et al., 2020). Na cidade de Sobral (CE) permanece a faixa etária de mulheres entre 20 a 39 anos, entre os anos de 2012 a 2017, com total de 336 casos dos 452 durante todo o período do estudo (MARQUES JVS, et al., 2018).

Em relação à escolaridade, o maior índice de gestantes com sífilis são as que não concluíram da $5^{\underline{a}}$ a $8^{\text {a }}$ série, tendo seu pico no ano de 2018, no qual o número de gestantes infectadas era 128 casos em relação a mulheres com nível superior incompleto e superior completo. No estudo apresentado por Sousa FDCA, et al. (2020), foi constatado que na cidade de Caxias (MA) cerca de $26,6 \%$ possuíam ensino fundamental incompleto, assim é notável que os dados comparam-se aos do município de Maceió.

De acordo com os resultados, o número e percentual de gestantes com sífilis, de acordo com sua raça/cor, o maior índice é nas mulheres de cor/raça parda, que tem seu maior valor em 2018 com o total de 276 (67,5\%) casos, tendo uma queda no ano de 2019 com 68 (59,1\%) casos. Entre os menores valores encontramos estão as mulheres de cor/raça amarela, que no ano de 2019 registrou $1(0,9 \%)$ caso. No entanto, Signor M, et al. (2018) ressalta que as características encontradas mostram que a ocorrência da sífilis não apresenta um perfil definido da população e varia conforme a região do Brasil. No Paraná, a partir dos dados encontrados, salienta-se a importância de ações preventivas com maior atenção para mulheres com baixa escolaridade.

Já o estudo de Pacheco VC, et al. (2018) identificou que, na cidade de Joinville (SC), as gestantes com sífilis identificadas de raça/cor branca são $84 \%$ dos casos e apenas $7,2 \%$ dos casos são em mulheres pardas no ano de 2015 , isso devido a maior parte da população da cidade ser considerada de raça/cor branca.

O tratamento mais utilizado na cidade de Maceió, segundo o estudo, é a Penicilina. Os dados revelam que entre os anos de 2015 a 2018, a medicação foi utilizada por mais de $80 \%$ dos casos. A penicilina é o fármaco de escolha no tratamento da sífilis gestacional e congênita, pois é uma medicação de fácil acesso e baixo 
custo (DALLÉ VC, et al., 2018). Porém, o número de gestantes que não realizou o tratamento aumentou de acordo com os anos do estudo, alcançando, em 2018, 4,4\% (18) dos casos. Um estudo realizado em Porto Velho (RO), entre 2009 e 2014 constatou que 65,15\% dos casos, também utilizaram a Penicilina para o tratamento da doença, mais uma vez pontuando que a medicação é a melhor escolha para tratar a gestante e o bebê (MOREIRA KFA, et al., 2017).

Os números e distribuição da sífilis segundo a idade da criança é maior entre os menores de 7 dias de idade, sendo importante destacar o rastreio da doença a partir das consultas de pré-natal. Entre os 2 aos 12 anos, não foram registrados casos de sífilis. No estudo de Guimarães TA, et al. (2018) o número e distribuição também é maior em crianças até 7 dias de idade. Os mesmos autores relatam que esse índice tem a ver com a forma que o tratamento tem ou não sido realizado, pois quando a mãe é adequadamente tratada com penicilina, o bebê absorve o medicamento através da placenta e nasce saudável.

As crianças com sífilis congênita têm em sua maioria o diagnóstico de sífilis congênita recente. Em 2019 foram $113(97,4 \%)$ casos. Já os casos de natimortos e abortos por sífilis vêm tendo uma queda desde 2014. Foi observado que na cidade de Recife (PE), os números de sífilis congênita equivalentes ao ano de 2018 tiveram uma taxa de $99 \%$ dos casos, contra $0,2 \%$ de natimortos e abortos por sífilis congênita (ROCHA JCX, et al., 2017)

Quando analisamos os casos de crianças com sífilis congênita, segundo informação sobre a realização do pré-natal pela mãe, temos um número maior entre as que realizaram as consultas. A partir do ano de 2014, das mulheres que realizaram o pré-natal foram $163(74,1 \%)$ contra $41(18,6 \%)$ que não. Porém, os números dos casos ignorados vêm crescendo nos anos estudados. Dados semelhantes aos encontrados por Lima VC, et al. (2017), que constataram $96,6 \%$ das gestantes realizaram o pré-natal e $62,1 \%$ das gestantes foram diagnosticadas durantes as consultas.

Em relação ao número e percentual da sífilis congênita e o esquema de tratamento da mãe, observamos que não tem sido adequado. No ano de 2018, a inadequação chegou a $136(63 \%)$ casos, contra $8(3,7 \%)$ de esquema adequado. Segundo Magalhães DMS, et al. (2013) o principal fator na falha do tratamento da sífilis na mãe está comumente relacionado a falta do tratamento do parceiro, pois o mesmo na maioria das vezes não é incluso ou não comparece as consultas de pré-natal, deixando de receber informações acerca da doença.

A pesquisa aponta que o número de casos de sífilis congênita segundo informações sobre tratamento do parceiro da mãe, no ano de 2019, apontou que 72 (72,1\%) casos não realizaram o tratamento contra 7 (6\%) que realizaram. No estado do Maranhão, entre os anos de 2014 a 2018, 59,9\% dos casos não realizaram o tratamento para a sífilis, contra $16,9 \%$ dos que realizaram.

Ficando clara a importância do incentivo ao acompanhamento do pai nas consultas de pré-natal e a realização do pré-natal do parceiro. Portanto, a assistência pré-natal apropriada colabora para a prevenção dos diversos desfechos adversos possíveis na gestação, além da diminuição dos gastos com a assistência ao recém-nascido, especialmente quando se trata de sífilis em gestantes que podem colaborar para o episódio de diversas sequelas no recém-nascido. (LEAL TLSL, et al., 2020).

\section{CONCLUSÃO}

Os achados desse estudo permitem concluir que o perfil epidemiológico da sífilis congênita em gestantes no município de Maceió ainda encontra-se com um elevado número de casos com 115 casos no ano de 2019, e maior parte entre mulheres diagnosticadas com a doença no $3^{\circ}$ trimestre, faixa etária de 20 a 29 anos, escolaridade entre $5^{\text {a }}$ a $8^{\text {a }}$ Série Incompleta, cor/raça parda, penicilina como principal método para 0 tratamento, tratamento, sífilis congênita recente como diagnóstico final da criança, tratamento da doença adequado e baixa adesão do parceiro para o seu tratamento. Diante disso, se faz necessário à busca ativa de gestantes para realização do pré-natal, e reforçar os exames clínicos entre as que já o realizam, assim como também o incentivo do acompanhamento às consultas pelos parceiros, e a realização do pré-natal do parceiro como preconizado pelo Ministério da Saúde. 


\section{REFERÊNCIAS}

1. BOTTURA BR, et al. Perfil epidemiológico da sífilis gestacional e congênita no Brasil. Período de 2007 a 2016. Arquivos Médicos dos Hospitais e da Faculdade de Ciências Médicas da Santa Casa de São Paulo, 2019; 64(2): 69-75.

2. BRASIL. Ministério da Saúde. Secretaria de Vigilância em Saúde. Programa Nacional de DST e AIDS. Boletim Epidemiológico Sífilis 2016. Brasília, 2016.

3. BRASIL. Ministério da Saúde. Secretaria de Vigilância em Saúde. Departamento de DST, Aids e Hepatites Virais. Boletim Epidemiológico HIV aids, 2017; 2(1).

4. BRASIL. Ministério da Saúde. Protocolo Clínico e Diretrizes Terapêuticas para Atenção Integral às Pessoas com Infecções Sexualmente Transmissíveis. Brasília: 2015a.

5. BRASIL. Ministério da Saúde. Boletim Epidemiológico - Sífilis, Brasília: 2015b.

6. BRASIL. Ministério da Saúde. Secretaria de Vigilância em Saúde. Departamento de Vigilância Epidemiológica. Doenças infecciosas e parasitárias: guia de bolso. Brasília: 2006.

7. BRASIL. Ministério da Saúde. Realização do Teste Rápido para HIV e Sífilis na atenção básica e aconselhamento em DST/Aids da Rede. Brasília: 2012.

8. BRASIL. Ministério da Saúde. Secretaria de Vigilância em Saúde. Departamento de DST, Aids e Hepatites Virais. Protocolo clínico e diretrizes terapêuticas para prevenção da transmissão vertical de HIV, sífilis e hepatites virais. Brasília: 2018.

9. CAVALCANTE PAM, et al. Sífilis gestacional e congênita em Palmas, Tocantins - 2007-2014. Epidemiologia e Serviços de Saúde, 2017; 26: 255-264.

10. DALLÉ J, et al. Dessensibilização oral à penicilina para o tratamento da sífilis na gestação: um exemplo de experiência bem-sucedida. Revista Brasileira de Ginecologia e Obstetrícia, 2018; 40(1): 43-46.

11. DE AQUINO GT, et al. Perfil das mulheres portadoras de sífilis gestacional em Santa Catarina no ano de 2012. Arquivos Catarinenses de Medicina, 2016; 44(4): 72-81.

12. GOMES FT, et al. Perfil epidemiológico dos casos de sífilis congênita no estado de Minas Gerais no período de 2007 a 2017. Scientia Plena, 2020; 16(3).

13. GUIMARÃES TA, et al. Sífilis em gestantes e sífilis congênita no Maranhão. Arquivos de Ciências da Saúde, 2018; 25(2): 24-30.

14. LEAL TLSL, et al. Perfil epidemiológico dos casos de sífilis congênita no Maranhão. Revista Eletrônica Acervo Científico, 2020; 8: e2936-e2936.

15. LIMA TM, et al. Perfil epidemiológico de pacientes com sífilis congênita e gestacional em um município do Estado de São Paulo, Brasil. Revista Brasileira de Saúde Materno Infantil, 2019; 19(4): 865-872.

16. LIMA VC, et al. Perfil epidemiológico dos casos de sífilis congênita em um município de médio porte no nordeste brasileiro. Journal of Health \& Biological Sciences, 2017; 5(1): 56-61.

17. MAGALHÃES DMS, et al. Sífilis materna e congênita: ainda um desafio. Cadernos de Saúde Pública, 2013; 29(6): 1109-1120.

18. MARQUES JVS, et al. Perfil epidemiológico da sífilis gestacional: clínica e evolução de 2012 a 2017. SANARE-Revista de Políticas Públicas, 2018; 17(2).

19. MOREIRA KFA, et al. Perfil dos casos notificados de sífilis congênita. Cogitare Enfermagem, 2017; 22(2).

20. NETO LG, et al. Epidemiologia da sífilis gestacional e congênita no estado de Goiás no período de 2013 a 2018. Revista Brasileira Militar de Ciências, 2019; 5(13).

21. PACHECO VC, et al. As influências da raça/cor nos desfechos obstétricos e neonatais desfavoráveis. Saúde em Debate, 2018; 42: 125-137.

22. PADOVANI C, et al. Sífilis na gestação: associação das características maternas e perinatais em região do sul do Brasil. Revista Latino-Americana de Enfermagem, 2018; 26.

23. ROCHA JCX, et al. Análise da Incidência e do Perfil Epidemiológico de Sífilis Congênita no Recife-PE. Revista SaúdeUNG-Ser, 2017; 10(no esp): 77.

24. SIGNOR M, et al. Spatial Distribution and Characterization of Cases of Congenital Syphilis. Journal of Nursing UFPE/Revista de Enfermagem UFPE, 2018; 12(2).

25. SOUSA FDCA, et al. Perfil da Sífilis na Gestação no Período de 2007/2016 em Caxias-MA. Revista Enfermagem Atual In Derme, 2020; 91(29). 